$\exists \int$ Journal of Logic \& Analysis 1:3 (2009) 1-22

ISSN 1759-9008

\title{
Integrals and valuations
}

\author{
THIERRY COQUAND \\ BAS SPITTERS
}

\begin{abstract}
We construct a homeomorphism between the compact regular locale of integrals on a Riesz space and the locale of measures(valuations) on its spectrum. In fact, we construct two geometric theories and show that they are biinterpretable. The constructions are elementary and tightly connected to the Riesz space structure.
\end{abstract}

2000 Mathematics Subject Classification 06D22, 28C05

Keywords: integration, locales, Riesz space/vector lattice, geometric logic

\section{Introduction}

The goal of this paper is to give a constructive formulation of the Riesz representation theorem. The Riesz representation theorem states that there is an isomorphism between integrals and regular measures on compact spaces. An integral on a compact Hausdorff space $X$ is a positive linear functional $I: C(X) \rightarrow \mathbb{R}$ (and we shall consider only maps such that $I(1)=1)$. A regular measure can be identified with a continuous valuation, where a valuation on $X$ is a map $\mu: O(X) \rightarrow[0,1]$ which is monotone, if $U \subseteq V$ then $\mu(U) \leqslant \mu(V)$, and such that $\mu(\emptyset)=0$ and $\mu(U \cap V)+\mu(U \cup V)=\mu(U)+\mu(V)$ and $\mu(X)=1$. The continuity condition demands that $\mu(U)$ is the sup of $\mu(V)$ for $V$ well-inside $U$ (i.e. such that $U$ contains the closure of $V$ ). An equivalent way to express this condition is to state the continuity property: if $V_{i}$ is a directed family then $\mu\left(\bigcup V_{i}\right)=\sup \mu\left(V_{i}\right)$. A subset is directed if it is inhabited and every two elements have a common upper bound. Such continuous valuations extend uniquely to Borel measures; see [1] for an overview.

From a constructive point of view there is a crucial difference between the two notions. We will now outline these differences; precise definitions can be found below. The integral $I(f)$ of a function $f \in C(X)$ is a Dedekind real. Intuitively, this means that one can compute arbitrary rational approximations. This may not be the case for the valuation $\mu(U)$ of an open $U$ : in general we do not have the property that for $r<s$,

$$
\mu(U)<s \vee r<\mu(U) .
$$


Constructively the valuation $\mu(U)$ is only a lower real, and can be thought of as a predicate $r<\mu(U)$ on the rationals. This predicate is downward closed: if $r<\mu(U)$ and $s \leq r$ then we have $s<\mu(U)$, but in general, given $\epsilon>0$ we are not given a way to compute a rational $\epsilon$ approximation of $\mu(U)$. Given an integral $I$ we define a corresponding valuation $\mu_{I}(U)$ by taking the sup of $I(f)$ over all $0 \leqslant f \leqslant 1$ the support of which is included in $U$. It is remarkable that for any valuation $\mu$ one can conversely find a (unique) integral $I$ such that $\mu=\mu_{I}$. So despite the fact that one may not be able to compute $\mu(U)$, it is still possible to compute $\int f d \mu$ as a Dedekind real as the supremum of

$$
\sum s_{i} \mu\left(s_{i}<f<s_{i+1}\right)
$$

over all partitions $s_{0}<\cdots<s_{n}$ of the range $f([a, b])$. A priori this supremum will only be a lower real.

As usual in constructive mathematics all structures carry a natural, but implicit, topology and all constructions are continuous. To make this structure explicit we start from a Riesz space $R$ and associate three formal spaces to it that are all compact completely regular: the maximal spectrum $\operatorname{Max}(R)=X$ (intuitively, $R$ is then a dense subset of $C(X)$ ), the space of integrals $\operatorname{INT}(R)$ and the space of valuations $\operatorname{VAL}(X)$. All three spaces are defined as propositional geometrical theories. A geometric formula is one of the form $\psi \Rightarrow \varphi$, where the formulas $\psi$ and $\varphi$ are positive, i.e. they are built up from atomic formulas using only (finite) conjunction, (infinite) disjunction. A geometric theory is a theory all of whose axioms are geometric. The main point of this paper is to define two interpretability maps, showing how to interpret the theory $\operatorname{VAL}(X)$ in the theory $\operatorname{INT}(R)$ (intuitively how to define the measure from an integral) and how to interpret the theory $\operatorname{INT}(R)$ in the theory $\operatorname{VAL}(X)$ (intuitively how to define the measure from the integral). The Riesz representation theorem can then be stated as the fact that these two maps define an homeomorphism between the corresponding formal spaces $\operatorname{VAL}(X)$ and $\operatorname{INT}(R)$ where the topology on $\operatorname{INT}(R)$ is the weak topology. Hence we arrive at a concrete constructive statement of the Riesz representation theorem which is valid in any topos.

The present article is part of our program to apply the logical approach to abstract algebra [7] to (functional) analysis [5, 9, 22, 6]. It may be seen as a contribution to Hilbert's program of logically translating the use of infinitary methods to finitary, or constructive, ones. It is also continuation of a tradition in topos theory, e.g. [2], but in a more explicit manner. ${ }^{1}$ It turns out that our program sometimes gives shorter proofs

\footnotetext{
${ }^{1}$ We avoid the axiom of (countable) choice, and, moreover, we refrain from using the power set axiom. One may wonder how we treat the set of all real numbers in such a framework. In fact, we do not use this set at all. We only consider the formal space of real numbers.
} 
of more general results than a direct constructive treatment in the sense of Bishop. Moreover, the space of valuations does not naturally carry a metric structure and hence the topological structure, explicit in our presentation, is hidden in Bishop's treatment of the Riesz representation theorem. We emphasize, however, that all our results are acceptable by Bishop's standard. Since we do not construct points, we have no use for the axiom of choice, not even the countable version which is available to Bishop.

\subsection{Formal measure and integration theory}

As outlined in $[4,22,8]$ a formal theory of measure and integration may be developed along the following lines.

In a usual set-theoretic foundation of measure theory one considers certain functions which are defined to be 'measurable'. Then relative to a measure one identifies all the functions which are equal almost everywhere and obtains a vector lattice $L_{0}$ of measurable 'functions'. Instead, one may consider such a vector lattice from the beginning, abstracting from the set-theoretic foundations. The benefits of this approach have been emphasized by Kolmogorov, Caratheodory and von Neumann [18]. In the present article we focus on the theory of integrals defined on formal functions and valuations defined on formal opens. For a formal treatment of Borel sets we refer to $[8,4,21]$.

The abstract space of functions is captured by a Riesz space (a vector lattice) which we require to have a strong unit ${ }^{2}$. An integral is a continuous linear functional on the Riesz space. On the other hand, a measure is typically only lower semi-continuous. This suggests that an integral will be a map to the Dedekind reals, but that a valuation will map to the lower reals. The Riesz representation theorem will be presented in the form of a homeomorphism between the formal space of integrals on a Riesz space and valuations on the opens of its spectrum. By the Stone-Yosida theorem any Riesz space $R$ with strong unit can be densely embedded in the space of continuous functions over its spectrum $\operatorname{Max}(R)$. This can be proved constructively [9]. The integral extends to this space of continuous functions. In this sense our approach is close to the Daniell integral.

Alternatively, we could have started from a compact completely regular locale $X$ and construct the Riesz space $C(X)$ of continuous functions on this locale. Then $\operatorname{Max}(C(X)) \cong X$. However, we also want to include 'syntactic' Riesz spaces such as the Riesz space of rational piecewise linear functions on $[0,1]$.

\footnotetext{
${ }^{2}$ An even weaker requirement would have been to demand that we are given a lattice ordered Abelian group. Such a group can be extended to a Riesz space over the rationals; e.g. [5].
} 
Thierry Coquand and Bas Spitters

\subsection{Overview}

Section 3 contains the statement of the Riesz representation theorem, the main result of the article. The statement is geometric with joins restricted to countable sets. This allows us to use logical methods to conclude classically that there has to be a constructive proof. We construct such a proof in Section 4. The proof uses a concrete theory of non-increasing functions, which we call $\Delta$-functions in Section 4.4.

\subsection{Notation}

We use the letters $a, b, f, g, \ldots$ for elements of $R$, and the letters $x, y, z, \ldots$ for elements of the lattice $\operatorname{Spec}(R)$. We write 1 for the top element of a lattice and 0 for the bottom element.

\section{Preliminaries}

\subsection{Various kinds of real numbers}

We recall some facts about the real numbers; see e.g. [14, D4.7].

Definition 1 A lower real is an inhabited, down-closed, open subset of the rationals. The collection of lower reals is denoted by $\mathbb{R}_{\text {low }}$. Upper reals are defined similarly and denoted $\mathbb{R}_{\text {up }}$. An interval consists of a pair $(L, U)$ of an upper real and a lower real such that $L \leqslant U$ : if $s$ in $L$ and $t$ in $U$, then $s<t$. A Dedekind real is an interval $(L, U)$ which is arbitrarily small: for every $s<t$, either $s \in L$ or $t \in U$. The Dedekind reals will be denoted by $\mathbb{R}$.

The lower reals include $+\infty$. In order to exclude it, we would need to pose a nongeometric restriction. This issue will not be important in the rest of the paper.

Lower (likewise upper) reals are closed under addition and closed under multiplication by a positive rational. The lower and upper reals are not closed under subtraction, but one can subtract a lower real from an upper real and obtain an upper real. The non-strict inequality $\leqslant$ is given by inclusion of subsets. The supremum of an inhabited set of lower reals is a lower real. The infimum of an inhabited set of upper reals is an upper real. This is an important motivation for the use of lower (upper) reals: the supremum of a sequence of rationals need not be a Dedekind real, but it is a lower real. 
In the absence of the powerset operator, the lower reals are better considered as a formal space rather than a set, but we will not emphasize this point.

In the presence of dependent choice the Dedekind reals coincide with the Cauchy reals.

Lemma 1 Let $L$ be a lower real and $U$ be an upper real.

If $L \leqslant U$, then for all rational $p, L+p-U \leqslant p$.

Conversely, if $L+p-U \leqslant p$ for some rational $p$, then $L \leqslant U$.

The following lemma will be used a number of times below:

Lemma 2 The relation $a \leqslant b+c$ for lower reals can be stated geometrically in two equivalent ways:

(1) $p<a \rightarrow \bigvee_{r+s=p}(r<b \wedge s<c)$;

(2) $r+s<a \rightarrow r<b \vee s<c$.

A similar statement holds for upper reals.

Proof The implication from 1 to 2 is direct. For the implication from 2 to 1 we observe that if $p<a$, then there exists $\varepsilon>0$ such that $p+\varepsilon<a$. Choose a rational $q<b$ and a natural number $N$ such that $p-q-N \varepsilon<c$. By hypothesis 2, with premiss $p+\varepsilon<a$, we have for every $n, q+n \varepsilon<b \vee p+\varepsilon-(q+n \varepsilon)<c$. Since the first disjunct holds for $n=0$ and the second for $n \geqslant N+1$, there exists $n$ such that $q+n \varepsilon<b$ and $p+\varepsilon-(q+(n+1) \varepsilon)<c$. We can now take $r=q+n \varepsilon$ and $s=p-q+n \varepsilon$.

The inequality between a lower real and a Dedekind real can also be stated geometrically.

\subsection{Logic and topology}

In set theory, i.e. in the topos Set, one uses topological spaces to deal with continuity. However, statements including points of topological spaces are often difficult to generalize to arbitrary toposes. Fortunately, it is often possible to resort to the lattice structure of the open sets of a topological space. These complete distributive lattices are thus called 'pointfree' spaces, or locales (see [13]). In the topos Set one can often reconstruct the points from this lattice; to be precise, there is an adjunction between the category 
of topological spaces and the category of locales, which restricts to an equivalence of categories between compact Hausdorff spaces and compact completely regular locales. In general, this equivalence is not present in a topos. When generalizing theorems from the topos Set to an arbitrary topos focusing on locales is often the better choice. One reason for this is that a locale may be defined by geometric theory. In logical terms the locale is its syntactic category, often called the Tarski-Lindenbaum algebra - that is, the poset of provable equivalence classes, ordered by provable entailment. The correspondence between the locale and the theory is the usual completeness and consistency link between theories and models. The models of the theory correspond to completely prime filters, i.e. points of the locale presented by the lattice. In this way, a point $x$ in a topological space defines a model of the corresponding theory: a basic proposition $I$ is true in the model $x$ iff $x \in I$. This view leads us to consider theories as primary objects of study; their models, the points, will be derived concepts. Hence topology is propositional geometric logic; see e.g. [14, 24].

\subsection{Spectrum of a Riesz space}

Definition $2 A n$ ordered vector space is a vector space with a partial order $\leqslant$ such that

(1) If $x \leqslant y$, then for all $z, x+z \leqslant y+z$;

(2) if $0 \leqslant x$, then for all $a \geqslant 0,0 \leqslant a x$.

A Riesz space (or vector lattice) is an ordered vector space where the order structure is a lattice. An element 1 is a strong unit if for all $x$ there exists $n$ such that $-n 1 \leqslant x \leqslant n 1$.

As noted in the introduction Riesz spaces provide an algebraic way to talk continuous functions on a compact completely regular locale.

We will consider Riesz spaces as $\mathbb{Q}$-vector spaces.

In a Riesz space one defines $f^{+}:=f \vee 0, f^{-}:=0 \vee(-f)$ and $|f|:=f^{+}+f^{-}$and derives that $f=f^{+}-f^{-}$.

The spectrum of a Riesz space $R$ is the space of all its representations - Riesz morphisms from $R$ to $\mathbb{R}$. It may be presented [9] as the locale which is freely generated by the collection of tokens $D(a)$, one for each $a$ in $R$, subject to the following relations:

1. $\quad D(1)=1$;

2. $D(a) \wedge D(-a)=0$; 
3. $D(a+b) \leqslant D(a) \vee D(b)$;

4. $D(a)=0$, if $a \leqslant 0$;

5. $D(a \vee b)=D(a) \vee D(b)$;

6. $D(a)=\bigvee_{s>0} D(a-s)$.

As proved in Proposition 2.6 in [5] one can derive the relations $D(a)=D\left(a^{+}\right)$and $D(a \wedge b)=D(a) \wedge D(b)$ from the ones above. In fact, either of them is equivalent to the relation 5 given (1-4).

One proves that this locale, $\operatorname{Max}(R)$, is compact and completely regular by interpreting the geometric theory (1-6) in the coherent theory (1-5) by interpreting $D(a)$ in (1-6) as $\bigvee_{s>0} D(a-s)$ in (1-5); see [5]. In terms of locales this means that the locale is a retraction of the coherent locale generated by (1-5). The relations for $\vee$ and $\wedge$ allow us to reduce $\vee \wedge D\left(a_{i j}\right)$ to $D\left(\vee \wedge a_{i j}\right)$. So the collection of $D(a)$ actually forms a basis, rather than only a subbasis, for the locale. We write $\operatorname{Spec}(R)$ for the distributive lattice generated by (1-5).

Theorem 1 [5] The order in $\operatorname{Spec}(R)$ is $D(a) \leqslant D(b)$ iff there exists $n$ such that $a^{+} \leqslant n b^{+}$. The order in the locale $\operatorname{Max}(R)$ is $D(a) \leqslant D(b)$ iff for all $\varepsilon>0$ there exists $n$ such that $(a-\varepsilon)^{+} \leqslant n b^{+}$.

The order on $\operatorname{Spec}(R)$, as opposed to the order on $\operatorname{Max}(R)$, is defined geometrically from the order on $R$.

Intuitively, the open $D(a)$ in the locale corresponds to the set $\{\sigma \mid \hat{a}(\sigma)>0\}$, where $\hat{a}: \operatorname{Max}(R) \rightarrow \mathbb{R}$ is the function defined by $\hat{a}(\sigma):=\sigma(a)$ for $\sigma$ in the locale. In the presence of the full axiom of choice this can be made precise as it allows us to prove that the spectrum has enough points.

Proposition 1 A model $m$ of the geometric theory above, i.e. a point of the spectrum as a locale, defines a representation

$$
\sigma_{m}(a):=(\{r \mid m \models D(a-r)\},\{s \mid m \models D(s-a)\}) .
$$

Proof Lemma 1 in [9] proves that this defines a Dedekind cut. By axiom $5 \sigma(a \vee b)=$ $\sigma(a) \vee \sigma(b)$. By axioms $2,4 D\left(1-\left(1-\frac{1}{n}\right)\right)=1$. It follows that $\sigma(1)=1$. As observed in [9] a map satisfying these properties is a representation.

The Stone-Yosida representation theorem states that there is a embedding of $R$ into the locale of (Dedekind) real valued continuous functions on its spectrum which is dense with respect to the sup-norm. The sup-norm is the upper real $\|a\|$ defined by $\|a\|<\lambda$ iff there exists $\lambda^{\prime}<\lambda$ such that $|a| \leqslant \lambda^{\prime} 1$. A constructive proof of this theorem can be found in [9]. 


\section{Statement of the Riesz-representation theorem}

The goal of this section is to state, in Subsection 3.4, the Riesz representation theorem as the existence of a homeomorphism between the formal compact completely regular spaces of integrals and valuations. Theorem 3 contains the proof of the representation theorem.

\subsection{The space of integrals}

Let $R$ be a Riesz space with strong unit 1 .

Definition $3 A$ (probability) integral $I$ on a Riesz space $R$ is a positive linear functional - that is, it is a linear map to the Dedekind reals and if $x \geqslant 0$, then $I(x) \geqslant 0$ - and such that $I(1)=1$.

An integral is continuous with respect to the sup-norm: if $|f| \leqslant r$, then $I(|f|) \leqslant r$, by positivity. By density of the Stone-Yosida embedding, an integral extends uniquely to a positive linear functional on the space of all continuous real-valued functions on the spectrum.

We present a geometric theory INT of integrals on $R$, much like the description of Stone's maximal spectrum $\operatorname{Max}(R)$ in section 2.3. In fact, the geometric theory Max will have one relation more than the theory INT. This means that INT can be interpreted in Max, this interpretation defines a frame map from INT to Max, and hence, a locale map from Max to INT. The locale Max is a sublocale of INT. The inclusion is given by assigning to a point its Dirac measure: $I_{x}(f):=f(x)$.

To wit, subbasic opens of INT, denoted by $[p<I(f)]$, are indexed by rational $p$ and $f$ in $R$. The set of its points will be $\{I \mid p<I(f)\}$. Since $p<I(f)$ iff $0<I(f-p)$, it is sufficient to treat basic opens of the form $0<I(f)$, written $P(f)$, where $P$ is a dummy symbol. The points in this open are integrals $I$ such that $0<I(f)$.

Definition 4 The geometric theory INT is freely generated by symbols $P(f), f$ in $R$, and relations:

I1 $P(1)=1$;

I2 $P(f) \wedge P(-f)=0$;

I3 $P(f+g) \leqslant P(f) \vee P(g)$; 
I4 $P(f)=0$, if $f \leqslant 0$;

Cont $P(f)=\bigvee_{s>0} P(f-s)$.

Lemma 3 The relation $P(f) \leqslant P(g)$ if $f \leqslant g$ holds in INT.

Proof $P(f-g+g) \leqslant P(f-g) \vee P(g)=0 \vee P(g)$.

As before one proves that INT is compact completely regular by reducing I1-4+Cont to I1-4. This result was proved by Coquand [5] who referred to the theory I1-4 as TOT, the theory of total orderings on an ordered vector space. We have chosen the present presentation of INT since it makes compact complete regularity easy to prove.

Lemma 4 The theory INT is equivalent to the theory of normalized positive additive functionals:

- $I(f) \in \mathbb{R}$

- $I(0)=0$;

- $I(f+g)=I(f)+I(g)$;

- $I(f) \geqslant 0$ if $f \geqslant 0$

- $I(1)=1$.

The notation above describes the locale with generators, $p<I(f)$ and $I(f)<q$, for $f$ in $R$ and $p, q$ rational and $I$ is a dummy symbol, and certain relations. For instance, the first axiom, $I(f) \in \mathbb{R}$, is a shorthand for the relations:

- $[p<I(f)] \leqslant\left[p^{\prime}<I(f)\right]$ if $p^{\prime}<p$;

- $[I(f)<q] \leqslant\left[I(f)<q^{\prime}\right]$ if $q<q^{\prime}$;

- $p<I(f)=\bigvee_{p^{\prime}>p} p^{\prime}<I(f)$;

- $I(f)<q=\bigvee_{q^{\prime}>q} q^{\prime}<I(f)$;

- $1=(p<I(f) \vee I(f)<q)$ if $p<q$;

- $0=(q<I(f) \wedge I(f)<p)$ if $p<q$.

Proof We interpret $P(f)$ in INT as $I(f)>0$ in the theory of positive additive functionals.

For the converse, we define $p<I(f)$ as $P(f-p)$ and $I(f)<q$ as $P(q-f)$. Then 
(1) $-\varepsilon<I(f)$ if $f \geqslant 0$ and $\varepsilon>0$. Proof: $P(f+\varepsilon-f) \leqslant P(f+\varepsilon) \vee P(-f)$ and $P(-f)=0$.

(2) $1=s<I(f) \vee I(f)<t$, whenever $s<t$. Proof: $P(t-s)=1$.

(3) By Lemma 3, if $s<I(f)$, then $t<I(f)$ for $t<s$. Similarly, if $I(f)<s$, then $I(f)<t$ for $s<t$.

Combined with the continuity rule, this shows that $I(f)$ is a Dedekind cut.

From I3 we have $P(f) \leqslant P\left(\frac{1}{n} f\right)$. Hence, $1-\frac{1}{n}<I(1)<1+\frac{1}{n}$, i.e. $I(1)=1$. Similarly, $I(0)<\frac{1}{n}$.

To prove additivity we combine Lemma 2 with $\mathbf{I 3}$ and obtain $I(f+g) \leqslant I(f)+I(g)$. Conversely, the rule $P(f) \wedge P(g) \leqslant P(f+g)$ can be derived in INT: $f=f+g-g$, so $P(f) \leqslant P(f+g) \vee P(-g)$ and the result follows from $P(g) \wedge P(-g)=0$.

Linearity readily follows from additivity, so the points of INT are integrals and, conversely, every integral defines a point.

Usually, one proves that the space of integrals is compact by an appeal to the Alaoglu theorem. Here we have shown that it is compact by construction. A similar construction can be carried out for Alaoglu's theorem for compact locales [17].

\subsection{Integrals on positive elements}

Instead of starting with a positive linear functional, it will later be convenient to work with its restriction to the positive elements.

Lemma 5 An integral is fixed by its behavior on the positive elements. As such it is a function $I: R^{+} \rightarrow \mathbb{R}^{+}$such that $I(0)=0$ and $I(f+g)=I(f)+I(g)$ and $I(1)=1$.

The theory of these functionals is geometric, we call this theory INTPOS.

Proposition 2 The geometric theories INT and INTPOS are biinterpretable.

Proof To obtain the integral from its positive part we define $I(f):=I\left(f^{+}\right)-I\left(f^{-}\right)$. 


\subsection{The space of valuations}

Definition 5 A valuation is a map $\mu: \operatorname{Spec}(R) \rightarrow \mathbb{R}_{\text {low }}^{+}$such that

- $\mu(0)=0, \mu(1)=1$;

- $\mu(x)+\mu(y)=\mu(x \vee y)+\mu(x \wedge y)$ (the modular law);

- If $x \leqslant y$ in $\operatorname{Spec}(R)$, then $\mu(x) \leqslant \mu(y)$ ( $\mu$ is monotone);

- $\mu(D(a)) \leqslant \bigvee_{\varepsilon>0} \mu(D(a-\varepsilon))$ Scott-continuous.

The theory of valuations is geometric, we call this theory VAL. Using Lemma 2 we can formulate modularity in a way similar to [16]. We have defined the valuation only on the coherent basis $\operatorname{Spec}(\mathrm{R})$ of $\operatorname{Max}(R)$, but it extends to the locale itself. Alternatively, we could have used the same definition but with monotonicity for the order on $\operatorname{Max}(R)$. This gives rise to the same locale of valuations: If $D(a) \leqslant D(b)$ in $\operatorname{Max}(R)$, then $D(a-r) \leqslant D(b)$ in $\operatorname{Spec}(R)$ for all $r>0$ and so $\mu(D(a-r)) \leqslant \mu(D(b))$. By Scott-continuity we get $\mu(D(a)) \leqslant \mu(D(b))$.

This locale coincides with the locale of valuations on the locale $\operatorname{Max}(R)$ as defined by Vickers [25] for an arbitrary locale with the difference that we require $\mu(1)=1$. Vickers [25, Prop.4.1] already pointed out that we can restrict to a base of the locale in order to obtain the locale of valuations geometrically from (a presentation) of the locale.

Classically, the regular measures form a compact Hausdorff space. Hence, classically, the locale of valuations on a compact completely regular locale is again compact completely regular. The homeomorphism in the Riesz representation theorem gives a constructive proof of this fact.

\subsection{Statement of the theorem}

We are now ready to define the promised maps between integrals and valuations. We give a syntactic bi-interpretation between two theories: the definition of the maps will be geometric, but the reasoning that these maps actually satisfy the required properties will be intuitionistic. For a general discussion of such techniques see e.g. [24, sec.4.5].

\section{From integrals to valuations}

Given an integral on a Riesz space, we construct a valuation on the opens in its spectrum:

$$
\mu_{I}(D(a)):=\sup \left\{I\left(n a^{+} \wedge 1\right) \mid n \in \mathbb{N}\right\}
$$


In section 4.5 we prove that this is well-defined, i.e. that it gives the same answer when $D(a)=D(b)$.

\section{From valuations to integrals}

In order to define the converse interpretation we introduce some notations. For $f$ in $R^{+}$define the lower real $\Delta_{f}(r, s):=\mu(r<f<s)$. Let $I=(r, s)$. Write $\Delta_{f}\left(I^{c}\right)$ for the lower real $\Delta_{f}(-\infty, r)+\Delta_{f}(s, \infty)$ and $\Delta_{f}[I]$ for the upper real $1-\Delta_{f}\left(I^{c}\right)$.

The interpretation of INTPOS in VAL

$$
\left.I_{\mu} f:=\operatorname{(sup}_{\left(s_{i}\right)} \sum s_{i} \Delta_{f}\left(s_{i}, s_{i+1}\right), \inf _{\left(s_{i}\right)} \sum s_{i+1} \Delta_{f}\left[s_{i}, s_{i+1}\right]\right)
$$

the $\left(s_{i}\right)$ range over partitions over a fixed interval $[a, b]$ where $a<f<b$. As is the case for $\mu_{-}$this is a disjunction over a concrete countable set: a finite list of strictly increasing rationals.

Assuming the classical Riesz representation theorem it is easy to show that these are indeed interpretations and that these maps are each other's inverses as follows: For any $r>0$ there is an $r$-approximation by sums $\sum s_{i} \Delta_{f}\left(s_{i}, s_{i+1}\right)$ and $\sum s_{i+1} \Delta_{f}\left[s_{i}, s_{i+1}\right]$. This follows from the usual classical proof of Riesz Theorem and the possibility to choose $s_{i}$ as continuity points for the function

$$
s \mapsto \Delta_{f}(-\infty, s)
$$

By completeness of propositional $\omega$-logic $[15,20]$ and the validity of the propositions in all models, i.e. measures or integrals, of the theory we see that, classically, there should be a proof in the theory that these are indeed interpretations. We will provide such a constructive proof in Theorem 3. This treatment is different from the classical one; see e.g. [19]. We take the topological/computational aspects into account by distinguishing between lower reals and Dedekind reals, moreover we do not use the extension of a valuation to a measure on the Borel sets. Our result is more general: not only is it constructive, and hence valid in any topos, but it also abstracts from a lattice of sets to a general lattice.

\section{Proof of the Riesz representation theorem}

\subsection{Formal simple functions}

We define formal simple functions on a distributive lattice $L$. All index sets in this section are finite, i.e. have a cardinality. We will use the convention that a capital letter, 
say $I$, is a subset of the variables indexed by the lower case letters, say $\left(x_{i}\right)$. For $\left(x_{i}\right)$ in $L$ we define $x_{I}:=\wedge\left\{x_{i} \mid i \in I\right\}$. Following Tarski [23] and Horn and Tarski [12, Def 1.4] we define the free monoid $M(L)$ such that the relation $x+y=x \vee y+x \wedge y$ holds. As Horn and Tarski prove this is the monoid of formal sums $\sum x_{i}$, where $x_{i}$ in $L$, with the following equality:

Lemma 6 [12] We have $\sum_{i \in I} a_{i}=\sum_{k \geqslant 1} \bigvee_{K \subset I,|K|=k} a_{K}$. Furthermore, $\sum_{i \in I} a_{i}=$ $\sum_{j \in J} b_{j}$ iff $\bigvee_{K \subset I,|K|=k} a_{K}=\bigvee_{K \subset J,|K|=k} b_{K}$ for all $k \geqslant 1$.

Definition 6 Let $M(L)$ be the monoid of formal sums in $L$ modulo the relation $x+y=x \vee y+x \wedge y$. We define the pre-order

$$
\sum x_{i} \leqslant \sum y_{j} \text { iff for all } I, x_{I} \leqslant \bigvee\left\{y_{J}|| J|=| I \mid\right\} .
$$

By Lemma $6 \leqslant$ is an order.

The monoid $M(L)$ satisfies the cancellation property; see [23]. For $k>0, k x \leqslant 0$ iff $x=0$. We add positive rational coefficients - that is, define a relation $\sum r_{i} x_{i} \leqslant \sum s_{j} y_{j}$ — by putting all the terms on one denominator. If $r$ in $\mathbb{Q}^{+}$and $x \leqslant y$, then $r x \leqslant r y$ and $x+z \leqslant y+z$. When $L$ is a lattice of sets, this coincides with the usual ordering of simple functions. We write $S^{+}(L)$ for the positive simple functions on $L$.

We write $r_{I}:=\sum_{i \in I} r_{i}$. The following is direct.

Lemma $7 \sum r_{i} x_{i} \leqslant \sum s_{j} y_{j}$ iff for all $I, x_{I} \leqslant \bigvee_{J, r_{I} \leqslant s_{J}} y_{J}$.

Lemma 8 The relation $\leqslant$ is transitive on $S^{+}(L)$.

Proof Suppose that $\sum r_{i} a_{i} \leqslant \sum s_{j} b_{j} \leqslant \sum t_{k} c_{k}$. By Lemma 6, for all $I, a_{I} \leqslant$ $\bigvee_{J, r_{I} \leqslant s_{J}} b_{J}$ and for all $J, b_{J} \leqslant \bigvee_{K, s_{J} \leqslant t_{K}} c_{K}$. So, $a_{I} \leqslant \bigvee_{J, K, r_{I} \leqslant s_{J}, s_{J} \leqslant t_{K}} c_{K}$.

\subsection{Extending valuations to simple functions}

We now consider the case where $L$ is $\operatorname{Spec}(R)$. We extend $\mu$ to an additive functional from the formal sums to the lower reals. This extension satisfies the modular law and hence so does the extension to the simple functions:

Lemma 9 If $\sum r_{i} x_{i} \leqslant \sum s_{j} y_{j}$, then $\mu\left(\sum r_{i} x_{i}\right) \leqslant \mu\left(\sum s_{j} y_{j}\right)$. So, $\mu$ is well-defined on $S^{+}(L)$ : if $k=l$, then $\mu(k)=\mu(l)$. 
Proof By bringing all the terms on one denominator we can dispose of all the scalars. Hence our goal will be to prove: If $\sum x_{i} \leqslant \sum y_{j}$, then $\mu\left(\sum x_{i}\right) \leqslant \mu\left(\sum y_{j}\right)$. To see this we have

$$
\mu\left(\sum x_{i}\right)=\mu\left(\sum_{k \geqslant 1} \bigvee_{|K|=k} x_{K}\right) \leqslant \mu\left(\sum_{k \geqslant 1} \bigvee_{|K|=k} y_{K}\right)=\mu\left(\sum y_{j}\right)
$$

Consider the dual lattice $L^{\prime}$ of $\operatorname{Spec}(R)$. We define $\mu(\neg x)$ as the upper real $1-$ $\mu(x)$. This definition is naturally extended to the formal simple functions $S^{+}\left(L^{\prime}\right)$ : $\mu\left(\sum s_{i}\left(\neg x_{i}\right)\right)=\left(\sum s_{i}\right)-\mu\left(\sum s_{i} x_{i}\right)$. However, we will not be able to define the valuation of a sum of mixed open and closed elements.

\subsection{Simple functions on the spectrum of a Riesz space}

We now consider the case where $L$ is the Boolean algebra freely generated by $\operatorname{Spec}(R)$. Let $f$ be in $R$. We denote the open $D(f-r)$ by $(f>r)$ and $D(r-f)$ by $(f<r)$ and the complement of $(f>r)$ by $(f \leqslant r)$ and the complement of $(f<r)$ by $(f \geqslant r)$.

We want to express the pointwise order relation between a positive simple function and a positive element of the Riesz space considered as continuous functions on the spectrum $\operatorname{Max}(R)$. However, for the sake of geometricity, we use the order of $\operatorname{Spec}(R)$ instead. Hence we are working with a coherent approximation to the pointwise order.

We define the relation $\sum r_{i} x_{i} \leqslant f$ as: for all $I, x_{I} \leqslant\left(r_{I} \leqslant f\right)$ and the relation $f \leqslant \sum s_{j} y_{j}$ as: $1=\bigvee_{J}\left(\left(f \leqslant s_{J}\right) \wedge y_{J}\right)$.

Lemma 10 If $a \leqslant 1$, then $a \leqslant D(a)$.

Proof We need to prove that $(a \leqslant 0) \vee((a \leqslant 1) \wedge D(a))=1$. We simplify this statement:

$(a>0) \leqslant(a \leqslant 1) \wedge D(a)$

$(a>0) \leqslant(a \leqslant 1)$

$(a>0) \wedge(a>1)=0$

The last statement follows from the hypothesis $a \leqslant 1$.

When $\operatorname{Max}(R)$ is spatial, as is the case in the presence of the axiom of choice, by StoneYosida, $f$ may be interpreted as a continuous function on $\operatorname{Max}(R)$ and the order above corresponds to a coherent approximation of the pointwise ordering of functions when the simple function $\sum r_{i} x_{i}$ is interpreted as the linear combination of the characteristic functions associated to the sets $x_{i}$. 
Lemma 11 Suppose that $\sum r_{i} x_{i} \leqslant \sum s_{j} y_{j}$ and $\sum s_{j} y_{j} \leqslant f$. Then $\sum r_{i} x_{i} \leqslant f$.

Proof We have $x_{I} \leqslant \bigvee_{J, r_{I} \leqslant s_{J}} y_{J}$ and $y_{J} \leqslant\left(s_{J} \leqslant f\right)$. So

$$
x_{I} \leqslant \bigvee_{J, r_{I} \leqslant s_{J}}\left(s_{J} \leqslant f\right) \leqslant \bigvee_{J, r_{I} \leqslant s_{J}}\left(r_{I} \leqslant f\right) \leqslant\left(r_{I} \leqslant f\right) .
$$

Lemma 12 Suppose that $f \leqslant \sum r_{i} x_{i}$ and $\sum r_{i} x_{i} \leqslant \sum s_{j} y_{j}$. Then $f \leqslant \sum s_{j} y_{j}$.

Proof We have $1=\bigvee_{I}\left(\left(f \leqslant r_{I}\right) \wedge x_{I}\right)$ and $x_{I} \leqslant \bigvee_{J, r_{I} \leqslant s_{J}} y_{J}$. So

$$
\begin{aligned}
1=\bigvee_{I}\left(\left(f \leqslant r_{I}\right) \wedge x_{I}\right) & =\bigvee_{I}\left(\left(f \leqslant r_{I}\right) \wedge \bigvee_{J(I), r_{I} \leqslant s_{J(I)}} y_{J(I)}\right) \\
& \leqslant \bigvee_{I} \bigvee_{J(I), r_{I} \leqslant s_{J(I)}}\left(f \leqslant s_{J(I)}\right) \wedge y_{J(I)} \leqslant \bigvee_{J}\left(\left(f \leqslant s_{J}\right) \wedge y_{J}\right) .
\end{aligned}
$$

It is clear that if $\sum r_{i} x_{i} \leqslant f \leqslant g$, then $\sum r_{i} x_{i} \leqslant g$, and if $f \leqslant g \leqslant \sum r_{i} x_{i}$, then $f \leqslant \sum r_{i} x_{i}$.

Lemma 13 If $\sum r_{i} x_{i} \leqslant f \leqslant \sum s_{j} y_{j}$, then $\sum r_{i} x_{i} \leqslant \sum s_{j} y_{j}$.

Proof We have for all $I, x_{I} \leqslant\left(r_{I} \leqslant f\right)$ and $1=\bigvee_{J}\left(\left(f \leqslant s_{J}\right) \wedge y_{J}\right)$. Then

$$
x_{I} \leqslant\left(r_{I} \leqslant f\right)=\left(r_{I} \leqslant f\right) \wedge \bigvee_{J}\left(\left(f \leqslant s_{J}\right) \wedge y_{J}\right) \leqslant \bigvee_{J, r_{I} \leqslant s_{J}} y_{J} .
$$

Lemma 14 Let $0 \leqslant f \leqslant b$ and let $s_{i}$ be a partition of $[0, b]$. Then

$$
\sum s_{i}\left(s_{i}<f<s_{i+1}\right) \leqslant f \leqslant \sum s_{i+1}\left(s_{i} \leqslant f \leqslant s_{i+1}\right) .
$$

Proof To prove the first inequality, we write $x_{i}:=\left(s_{i}<f<s_{i+1}\right)$. The $x_{i}$ are disjoint and

$$
x_{i} \leqslant\left(s_{i}<f\right) \leqslant\left(s_{i} \leqslant f\right) .
$$

To prove the second inequality we write $t_{i}:=\left(s_{i} \leqslant f \leqslant s_{i+1}\right)$. Then $\left(f \leqslant s_{i+1}\right) \wedge y_{i}=y_{i}$ and $1=\bigvee y_{i}$, since $s_{0}, \ldots, s_{n}$ is a partition of $[0, b]$.

The following results have a direct proof. 
Lemma 15 If $l_{1} \leqslant f_{1}$ and $l_{2} \leqslant f_{2}$, then $l_{1}+l_{2} \leqslant f_{1}+f_{2}$. Similarly, if $f_{1} \leqslant k_{1}$ and $f_{2} \leqslant k_{2}$, then $f_{1}+f_{2} \leqslant k_{1}+k_{2}$.

The spectrum of a Riesz space is completely regular as the following simple formulation of the Urysohn's Lemma shows.

Lemma 16 Let $a$ in $R^{+}$and $\varepsilon>0$. Then $D(a-\varepsilon) \leqslant \frac{1}{\varepsilon}(a \wedge \varepsilon) \leqslant D(a)$.

Proof For the first inequality we need to prove that $D(a-\varepsilon) \leqslant\left(1 \leqslant \frac{1}{\varepsilon}(a \wedge \varepsilon)\right)$. Since the right hand side is a formal complement this means, $D(a-\varepsilon) \wedge\left(1>\frac{1}{\varepsilon}(a \wedge \varepsilon)\right)=0$. Now, $\left(1>\frac{1}{\varepsilon}(a \wedge \varepsilon)\right)=(\varepsilon>a)=D(\varepsilon-a)$.

The second inequality follows from Lemma 10: $\frac{1}{\varepsilon}(a \wedge \varepsilon) \leqslant D\left(\frac{1}{\varepsilon}(a \wedge \varepsilon)\right)=D(a)$.

\section{4 $\Delta$-functions}

In this subsection we fix $f \geqslant 0$ in $R$ and a valuation $\mu$. We define the lower real $\Delta(r, s)=\mu(r<f<s)$ and the upper real $\Delta[r, s]=1-\Delta(-\infty, r)-\Delta(s, \infty)$ as in Section 3.4. Intuitively, the function $\Delta$ represents the function $\alpha(s)=\mu(f<s)$ which is used in the definition of the integral as a Stieltjes integral $\int f \mathrm{~d} \mu=\int s \mathrm{~d} \alpha(s)$. The functions $\Delta$ satisfies:

(1) $\Delta(0, b)=1$ for some $b$;

(2) $\Delta(r, s) \leqslant 1$;

(3) $\Delta(r, s) \geqslant 0$;

(4) $\Delta(r, s)+\Delta(s, t)=\Delta(r, t)-\Delta[s]$

(5) $\Delta\left(r^{\prime}, s^{\prime}\right) \leqslant \Delta(r, s)$ whenever $r \leqslant r^{\prime}<s^{\prime} \leqslant s$;

(6) $\Delta\left(r, s^{\prime}\right)+\Delta\left(r^{\prime}, s\right)=\Delta(r, s)+\Delta\left(r^{\prime}, s^{\prime}\right)$ whenever $r<r^{\prime}<s^{\prime}<s$;

(7) $\Delta(r, s)=\bigvee\left\{\Delta\left(r^{\prime}, s^{\prime}\right) \mid r<r^{\prime}<s^{\prime}<s\right\}$.

In $4, \Delta[s]:=\Delta[s, s]$.

We write $\left(r^{\prime}, s^{\prime}\right) \ll(r, s)$ for $r<r^{\prime}<s^{\prime}<s$. As before, we write $\Delta(I)$ for $\Delta(r, s)$, if $I=(r, s)$.

Lemma 17 If $I \ll J$ and $p<q$, then $\Delta(J)>p$ or $\Delta[I]<q$.

Proof Since $\Delta\left(I^{c}\right)+\Delta(J) \geqslant 1>p+(1-q)$. 
We now prove 'a non-increasing function is continuous in a dense set of points' in a pointfree way.

Theorem 2 Let $N \in \mathbb{N}$ and $I=(r, s)$ be an open interval. Then there exists an interval $J \ll I$ such that $\Delta[J]<\frac{1}{N}$.

Proof Choose $2 N$ disjoint intervals $I_{i}$ in $I$ and choose $2 N$ intervals $J_{i} \ll I_{i}$. For each $i, \Delta\left(I_{i}\right)>\frac{1}{2 N}$ or $\Delta\left[J_{i}\right]<\frac{1}{N}$. It is impossible that the former case occurs all the time, therefore the latter case occurs at least once.

It follows classically that $\mu(r<f \leqslant s)=\inf _{s^{\prime}>s} \mu\left(r<f<s^{\prime}\right)$. The approximations to this infimum are explicit in the following proposition which assigns a Dedekind real to $\Delta$. The interpretation of this real is the Stieltjes integral $\int s \mathrm{~d} \alpha(s)$, where $\alpha$ is a non-decreasing function connected to $\Delta$.

\section{Proposition 3 The pair}

$$
\left(\left\{p \mid p<\sum s_{i} \Delta\left(s_{i}, s_{i+1}\right)\right\},\left\{q \mid \sum s_{i+1} \Delta\left[s_{i}, s_{i+1}\right]<q\right\}\right),
$$

where $s_{i}$ ranges over finite partitions of $[0, b]$, defines a Dedekind real.

Proof We first prove that the upper and lower cut come arbitrary close: There exists $\left(s_{i}\right)$ such that $\sum s_{i+1} \Delta\left[s_{i}, s_{i+1}\right]-\sum s_{i} \Delta\left(s_{i}, s_{i+1}\right)$ is small. To wit, given $\varepsilon>0$, use Theorem 2 to choose a partition $s_{i}$ of $[a, b]$ such that $\left|s_{i+1}-s_{i}\right|<\varepsilon$ and $\sum \Delta\left[s_{i}\right]<\varepsilon$. Then

$$
\begin{aligned}
\sum s_{i+1} \Delta\left[s_{i}, s_{i+1}\right] & -\sum s_{i} \Delta\left(s_{i}, s_{i+1}\right) \\
& \leqslant \sum\left(s_{i+1}-s_{i}\right) \Delta\left[s_{i}, s_{i+1}\right]+\sum s_{i}\left(\Delta\left[s_{i}\right]+\Delta\left[s_{i+1}\right]\right) \\
& \leqslant \varepsilon \sum \Delta\left[s_{i}, s_{i+1}\right]+2 b \varepsilon \\
& \leqslant \varepsilon\left(1+\sum \Delta\left[s_{i}\right]\right)+2 b \varepsilon \leqslant \varepsilon(1+\varepsilon)+2 b \varepsilon .
\end{aligned}
$$

We now prove that the lower cut is below the upper cut. By Lemma 14,

$$
l:=\sum s_{i}\left(s_{i}<f<s_{i+1}\right) \leqslant f \leqslant \sum s_{i+1}\left(s_{i} \leqslant f \leqslant s_{i+1}\right)=: k .
$$

Write $y_{i}:=\left(f<s_{i}\right) \vee\left(s_{i+1}<f\right)$. By Lemma 13, $l+\sum s_{i+1} y_{j} \leqslant \sum s_{i+1}$, so $\mu(l)+$ $\mu\left(\sum s_{i+1} y_{j}\right) \leqslant \mu\left(\sum s_{i+1}\right)$. The conclusion, $\mu(l) \leqslant \mu(k)$, follows from Lemma 1 .

The previous proposition contains the essence of Bishop's profile theorem; see [3]. It is the crucial step in the proof that $I_{\mu}$ is a function; see Lemma 19. 


\subsection{Continuous maps}

We are now ready to show that the maps $\mu_{I}$ and $I_{\mu}$ defined above indeed map integrals to valuations, and vice versa. We need to check that the interpretations of all the axioms hold.

We first repeat the definition from section 3.4:

$$
\mu_{I}(D(a)):=\sup \left\{I\left(n a^{+} \wedge 1\right) \mid n \in \mathbb{N}\right\} .
$$

We observe that $f \leqslant D(a)$ iff there exists $n$ such that $f \leqslant n a^{+} \wedge 1$. Hence, $\sup \left\{I\left(n a^{+} \wedge 1\right) \mid n \in \mathbb{N}\right\}=\sup \{I(f) \mid f \leqslant D(a)\}$.

The map $\mu_{I}$ extends to the positive simple functions:

$$
\mu_{I}\left(\sum r_{i} D\left(a_{i}\right)\right)=\sup \left\{I\left(\sum r_{i}\left(n a_{i}^{+} \wedge 1\right)\right) \mid n \in \mathbb{N}\right\} .
$$

Lemma $18 \mu_{I}$ is a valuation.

Proof To prove modularity we observe that

$$
(n a \wedge 1)+(n b \wedge 1)=(n(a \wedge b) \wedge 1)+(n(a \vee b) \wedge 1)
$$

and hence

$$
I(n a \wedge 1)+I(n b \wedge 1)=I(n(a \wedge b) \wedge 1)+I(n(a \vee b) \wedge 1) .
$$

For monotonicity: If $f \leqslant x$ and $x \leqslant y$, then $f \leqslant y$. Finally, regularity, $\mu(D(a))=$ $\sup _{r>0} \mu(D(a-r))$ is direct.

We generalize the definition of $I_{\mu}$ in section 3.4 to arbitrary simple positive functions:

$$
I_{\mu}(f)=\left(\sup \left\{\mu(l) \mid l \leqslant f, l \in S^{+}(L)\right\}, \inf \left\{\mu(k) \mid f \leqslant k, k \in S^{+}\left(L^{\prime}\right)\right\}\right) .
$$

We will prove that the supremum and the infimum over the restricted sets of simple functions used in section 3.4 already form a Dedekind real and hence the two definitions coincide.

Lemma $19 I_{\mu}$ is an integral.

Proof To prove that $I$ maps to the Dedekind reals: Let $f \in R^{+}$and choose $b \geqslant f$. By Proposition $3\left(\sup \sum s_{i} \Delta\left(s_{i}, s_{i+1}\right)\right.$, inf $\left.\sum s_{i+1} \Delta\left[s_{i}, s_{i+1}\right]\right)$ is a Dedekind real: the lower cut is below the upper cut and both cuts 'kiss'.

To prove additivity, by Lemma 15 , if $l_{1} \leqslant f$ and $l_{2} \leqslant g$, then $l_{1}+l_{2} \leqslant f+g$. Hence $I(f)+I(g) \leqslant I(f+g)$. Conversely, if $f \leqslant k$ and $g \leqslant l$, then $f+g \leqslant k+l$ and hence $I(f+g) \leqslant I(f)+I(g)$. 


\subsection{Homeomorphism}

We prove that there is a homeomorphism between the integrals on a Riesz space and the valuations on the opens of the spectrum.

Theorem 3 [Riesz representation theorem] Let $R$ be a Riesz space with a strong unit. The theory of valuations on its spectrum is equivalent to the theory of integrals on $R$. It follows that the corresponding compact completely regular locales are homeomorphic.

Proof That is, we claim that $I_{\mu_{J}}=J$ and $\mu_{I_{\nu}}=\nu$.

$$
\begin{aligned}
I_{\mu_{J}}(f) & =\sup \left(\left\{\mu_{J}(l) \mid l \leqslant f\right\}\right) \\
& =\sup \{J(g) \mid g \leqslant l \leqslant f\} \\
& \geqslant \sup \{J(f-\varepsilon) \mid \varepsilon>0\}=J(f)
\end{aligned}
$$

For the inequality we observe that for each $\varepsilon>0, f-\varepsilon \leqslant \sum_{n \geqslant 1} \frac{\varepsilon}{2}\left(\left(n+\frac{1}{2}\right) \frac{\varepsilon}{2}<f\right) \leqslant f$. The other inequality is trivial.

Conversely,

$$
\begin{aligned}
\mu_{I_{\nu}}(k) & =\sup \left\{I_{\nu}(f) \mid f \leqslant k\right\} \\
& =\sup \{\nu(l) \mid l \leqslant f \leqslant k\} \\
& \geqslant \sup \{\nu(l) \mid l \ll k\}=\nu(k)
\end{aligned}
$$

Where $l \ll k$ means $k=\sum s_{i} D\left(a_{i}\right)$ and $l=\sum s_{i} D\left(a_{i}-\varepsilon\right)$. By the Urysohn Lemma 16 there exists $f$ in $R$ such that $l \leqslant f \leqslant k$. The other inequality is trivial.

\section{Related work}

Vickers [25] presents another variant of the Riesz representation theorem. His construction works for locales which are not necessarily compact completely regular. However, his integrals have their values in the lower (or upper) reals, as opposed to the Dedekind reals. A locale of valuations was first presented by Heckman [10].

The present homeomorphism has already been applied in a non-commutative context of quantum theory [11] where it provides an isomorphism between quasi-states and certain valuations. Quasi-states are used in the algebraic foundations of quantum mechanics. 
Thierry Coquand and Bas Spitters

\section{Conclusions}

The present construction was motivated by Bishop's bijection between measures and integrals [3]. Bishop's forces the measure of a measurable set to be a Dedekind real. This is somewhat inconvenient in practice since for a measurable function $f$ the measure of $[f \geqslant s]$ need not be Dedekind in general. We believe that the present theory allows for a smoother development of, at least, the abstract functional analytic aspects of Bishop's measure theory.

\section{Acknowledgements}

We would like to thank Alex Simpson, Steve Vickers and the referees for comments on the presentation of this paper.

\section{References}

[1] M Alvarez-Manilla, A Jung, K Keimel, The probabilistic powerdomain for stably compact spaces, Theoret. Comput. Sci. 328 (2004) 221-244; doi: 10.1016/j.tcs.2004.06.021.

[2] B Banaschewski, C J Mulvey, A globalisation of the Gelfand duality theorem, Annals of Pure and Applied Logic 137 (2006) 62-103; doi: 10.1016/j.apal.2005.05.018.

[3] E Bishop, D Bridges, Constructive analysis, volume 279 of Grundlehren der Mathematischen Wissenschaften, Springer-Verlag (1985).

[4] T Coquand, A note on measures with values in a partially ordered vector space, Positivity 8 (2004) 395-400; doi:10.1007/s11117-004-7399-0.

[5] T Coquand, About Stone's Notion of Spectrum, Journal of Pure and Applied Algebra 197 (2005) 141-158; doi: 10.1016/j.jpaa.2004.08.024.

[6] T Coquand, Geometric Hahn-Banach theorem, Math. Proc. Cambridge Philos. Soc. 140 (2006) 313-315; doi: 10.1017/S0305004105008935.

[7] T Coquand, H Lombardi, A logical approach to abstract algebra, Math. Structures Comput. Sci. 16 (2006) 885-900; doi: 10.1017/S0960129506005627.

[8] T Coquand, E Palmgren, Metric Boolean algebras and constructive measure theory, Arch. Math. Logic 41 (2002) 687-704; doi: 10.1007/s001530100123.

[9] T Coquand, B Spitters, Formal Topology and Constructive Mathematics: the Gelfand and Stone-Yosida Representation Theorems, Journal of Universal Computer Science 11 (2005) 1932-1944. 
[10] R Heckmann, Probabilistic power domains, information systems, and locales, from: "Mathematical Foundations of Programming Semantics VIII, volume 802 of Lecture Notes in Computer Science", Springer Verlag (1994) 410-437.

[11] C Heunen, K Landsman, B Spitters, A topos presentation of algebraic quantum theory (2008); arXiv:0709.4364.

[12] A Horn, A Tarski, Measures in Boolean algebras, Trans. Amer. Math. Soc. 64 (1948); doi: $10.2307 / 1990396$.

[13] P T Johnstone, Stone Spaces, Cambridge University Press (1982).

[14] P T Johnstone, Sketches of an Elephant: A topos theory compendium, volume 2, Clarendon Press (2002).

[15] M Makkai, GE Reyes, First order categorical logic, Springer-Verlag, Berlin (1977)Model-theoretical methods in the theory of topoi and related categories, Lecture Notes in Mathematics, Vol. 611; doi: 10.1016/0001-8708(78)90068-3.

[16] MA Moshier, A Jung, A Logic for Probabilities in Semantics, from: "CSL '02: Proceedings of the 16th International Workshop and 11th Annual Conference of the EACSL on Computer Science Logic", Springer-Verlag (2002) 216-231; doi:10.1007/3540-45793-3_15.

[17] C J Mulvey, J W Pelletier, The dual locale of a seminormed space, Cahiers Topologie Géom. Différentielle 23 (1982) 73-92Third Colloquium on Categories, Part VI (Amiens, 1980).

[18] G-C Rota, Twelve problems in probability no one likes to bring up, from: "Algebraic combinatorics and computer science", Springer Italia, Milan (2001) 57-93.

[19] W Rudin, Real and complex analysis, third edition, McGraw-Hill Book Co., New York (1987).

[20] D Scott, A Tarski, The sentential calculus with infinitely long expressions, Colloq. Math. 6 (1958) 165-170; doi: 10.2307/2270592.

[21] A Simpson, Formal Borel sets - a proof-theoretic approach (2007)Joint LICS/Logic Colloquium talk, Wroclaw, Poland.

[22] B Spitters, Constructive algebraic integration theory without choice, from: "Mathematics, Algorithms, Proofs", (T Coquand, H Lombardi, M-F Roy, editors), Dagstuhl Seminar Proceedings 05021, Internationales Begegnungs- und Forschungszentrum (IBFI), Schloss Dagstuhl, Germany (2005).

[23] A Tarski, Algebraische Fassung des Massproblems, Fund. Math. 31 (1938) 47-66.

[24] S Vickers, Locales and Toposes as Spaces, from: "Handbook of Spatial Logics", (M Aiello, IE Pratt-Hartmann, JF van Benthem, editors), Springer (2007); doi: 10.1007/978-1-4020-5587-4_8.

[25] S Vickers, A Localic Theory of Lower and Upper Integrals, Mathematical Logic Quarterly 54 (2008) 109-123; doi: 10.1002/malq.200710028. 
Computing Science Department, Göteborg University

Mathematics Department, Eindhoven University of Technology

coquand@chalmers.se, spitters@cs.ru.nl

Received: 15 May 2008 Revised: 17 March 2009 